\title{
Analysis and Control on a Specific Electromagnetic Micromirror
}

\author{
Wanyan Sun, ${ }^{1,2}$ Yonghong Tan $\left(\mathbb{D},{ }^{3}\right.$ Ruili Dong, ${ }^{4}$ and Wenjiang Shen ${ }^{5}$ \\ ${ }^{1}$ Tianjin Key Laboratory of Intelligent Robotics, Nankai University, Tianjin 300350, China \\ ${ }^{2}$ Institute of Robotics and Automatic Information System, College of Artificial Intelligence, Nankai University, \\ Tianjin 300350, China \\ ${ }^{3}$ College of Information Mechanical and Electrical Engineering, Shanghai Normal University, Shanghai 201418, China \\ ${ }^{4}$ College of Information Science and Technology, Donghua University, Shanghai 200000, China \\ ${ }^{5}$ Suzhou Institute of Nano-tech and Nano-bionics, Chinese Academy of Science, Suzhou 215123, China
}

Correspondence should be addressed to Yonghong Tan; tany@shnu.edu.cn

Received 1 November 2019; Revised 27 June 2020; Accepted 28 August 2020; Published 10 September 2020

Academic Editor: Ewa Pawluszewicz

Copyright $\odot 2020$ Wanyan Sun et al. This is an open access article distributed under the Creative Commons Attribution License, which permits unrestricted use, distribution, and reproduction in any medium, provided the original work is properly cited.

In this paper, 10 resonant modes were divided based on the structure of a specific two-dimensional electromagnetic micromirror from Professor Shen's team and Finite Element Method (FEM), and using as many as 10 resonant modes to do such analysis was the first time according to the best of our knowledge. The results showed that the slow axis can participate in several resonant motions under the signals of resonant frequencies. In particular, participating in Mode 1 for slow-scan axis was the key reason to the instability of Vertical Refreshing Scanning (VRS) in raster scanning. In addition, a piecewise PID control based on filters design for this electromagnetic micromirror was proposed to suppress the unexpected resonant oscillation and to improve the angular positioning accuracy in slow-scan axis control. Finally, the proposed method was applied to electromagnetic micromirror stages, and the experimental results showed that the proposed approach was reliable.

\section{Introduction}

Micromirror is a MEMS (Micro-Electro-Mechanical System) device, which is widely used in optical communication, head-worn display, LIDAR (Lighting Detection and Ranging), projector, and medical imaging detection [1-9]. There are four main types of micromirrors in driving manners, including in electrostatic micromirror $[3,10]$, piezoelectric micromirror [11], electro-thermal micromirror [12], and electromagnetic micromirror [5, 6]. Among them, electrostatic micromirror is well developed for use by Thomas Bifano and Texas Instrument Inc. Thomas Bifano reported an array of electrostatic actuator shaping the mirror [4], while Texas Instrument Inc. was dedicated to developing an array of aluminum micrometer-sized mirrors, in an orthogonal layout for full HD projector and display [13]. Electromagnetic micromirrors are outstandingly of many advantages, such as lower power consumption, smaller size, and larger deflection angle $[7,14,15]$. So an electromagnetic micromirror was chosen to discuss in this paper.
Ji et al. [16] proposed a two-dimensional electromagnetic micromirror using single turn coil and radial magnetic field from concentric magnet assembly in 2007, which was the first paper reported on this topic. Design and fabrication of this electromagnetic micromirror and 6 vibration modes were focused on in their paper, and the control was located in open loop control. Chen et al. [5] reported a two-dimensional scanning micromirror electromagnetically driven for slow-scan axis and harmonically driven for the fast-scan axis with larger mechanical angles up to $\pm 7.5^{\circ}$ and $\pm 12.5^{\circ}$, respectively. Structure and fabrication of the electromagnetic micromirror and the sensitivity of piezo-resistive sensor were emphasized to study, but control is still in open loop control. Steve et al. [17] focused on Newton's method to determine the harmonic coefficients of the two-dimensional electromagnetic micromirror, but controlling the mirror deflection to correctly produce the desired angular motion presented a significant engineering challenge. Han et al. [18] proposed a new electromagnetic micromirror of unique current paths and gimbal geometry to provide independent 
2-DOF (degree-of-freedom) actuation capability and large mechanical half scan angle up to $\pm 6.43^{\circ}$ and $\pm 4.2^{\circ}$, and PID control and a low-pass filter (LPF) were used in their research. However, the rings from driving coils of two axes were difficult to be eliminated.

Therefore, based on the structure of an electromagnetic micromirror supported by patents [19-22] from Prof. Wenjiang Shen's team, 10 resonant modes were divided to estimate the resonant frequencies for the first time in this paper. The results showed that the slow axis participated in several resonant motions under the signals of resonant frequencies, which were the key reasons for the instability in slow-scan axis control of this two-dimensional electromagnetic micromirror. Modal analysis of this electromagnetic micromirror was presented, and resonant frequencies were acquired. Then, a piecewise PID controller with filters was designed to improve the performances of this instability. Finally, the proposed methods were applied to an electromagnetic micromirror platform to determine their reliability.

\section{Modal Analysis of Electromagnetic Micromirror}

Structure of this electromagnetic micromirror is shown in Figure 1. The inner frame is connected to the outer frame through slow beams (Beam 1 and Beam 2). Ellipse micromirror and ellipse reinforce ring are connected to the inner frame through quick beams (Beam 3 and Beam 4). Four-Terminal Piezo-resistive Sensors (FTPSs) [23] are designed at the end of Beam 2 and Beam 4, respectively. In the beginning, a magnetic flux density $B$ is applied externally with $45^{\circ}$ to both the quick beams and the slow beams. Meanwhile, the beams, outer frame, inner frame, ellipse reinforce ring, ellipse micromirror, and FTPSs are in the same plane. Quick beams are perpendicular to slow beams. Beam 1 and Beam 2, Beam 3 and Beam 4 are symmetrical, respectively, and they are not in torsion. $m$ turns of DACs (Driving Au Coils) are formed elliptically on the inner frame to drive this electromagnetic micromirror by current flow. Since current flow in a wire results from motion of charges in this wire, a wire with current placed in a magnetic field where directions of current and electromagnetic cannot parallel exerts magnetic forces. For a differential length $d l$, of a wire of current $\vec{I}$ placed in a magnetic field $\vec{B}$, the force $d \vec{F}$ [24] is given by

$$
d \vec{F}=\vec{I} d l \times d \vec{B},
$$

where $\times$ is a cross product. Combining with (1), an electromagnetic torque $T_{e}$ that DACs generate under $\vec{B}$ is shown as follows:

$$
\begin{aligned}
T_{e} & =\sum_{j_{1}=1}^{m} \oint_{L_{j_{1}}} \vec{r}_{j_{1}} \times d \vec{F}_{j_{1}}=\sum_{j_{1}=1}^{m} \oint_{L_{j_{1}}} \vec{r}_{j_{1}} \times \vec{I} d l_{j_{1}} \times \vec{B} \\
& =\sum_{j_{1}=1}^{m} \oint_{L_{j_{1}}} r_{j_{1}} I d l_{j_{1}} B \sin \theta=B I \sum_{j_{1}=1}^{m} \oint_{L_{j_{1}}} r_{j_{1}} d l_{j_{1}} \sin \theta,
\end{aligned}
$$

where $\vec{r}_{j_{1}}$ is the distance between unit length of the coil and the rotation axis on the $j_{1}$ th coil $\left(j_{1}=1, \ldots, m\right)$, the

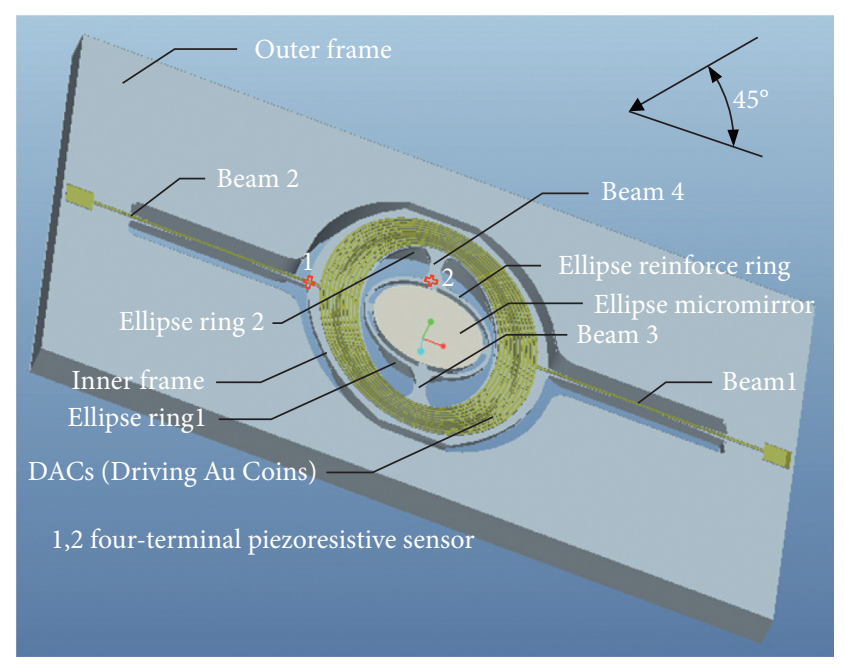

FIgURE 1: Structure of electromagnetic micromirror.

innermost elliptical Au coil is the first coil, and the outermost elliptical Au coil is the $m$ th coil. $L_{j_{1}}$ is the closed path where current is running through on the $j_{1}$ th coil $\left(j_{1}=1, \ldots, m\right)$, and $\theta$ is the angle between $\vec{I}$ and $\vec{B}$. Elliptical ring 1 and elliptical ring 2 are under ellipse micromirror and inner frame, respectively.

Based on this structure (Figure 1) together with the main properties of this electromagnetic micromirror shown in Table 1, 10 resonant modes (Figure 2) are divided through ANSYS Workbench [18] in FEM, and the characters of modal analysis are shown in Table 2, which shows a detailed description of the 10 kinds of movements corresponding to Figure 2.

Among motions in Figure 2, Mode 1 and Mode 6 are usually used in Lissajous scanning as main operations, but they are hard to be controlled [13]. Different from Lissajous scanning, in this paper, raster scanning [1] is applied (Figure 3), which can avoid rings caused by driving coils for the fast axis and the slow axis. Sine is a signal of single frequency. Any signal, such as triangular waves, step signals, and so on, can be thought of as a composite of many sine signals of different amplitudes and frequencies [25]. If these signals of different frequencies as the input signals inspired the resonant motions shown in Figure 2, the triggered ringing effects could also occur. To avoid this phenomenon, sine signal is chosen as the input voltage. Sine signal of a high frequency in the horizontal direction called Horizontal Fast Scanning (HFS) is given; meanwhile, sine signal of a low frequency in the vertical direction called Vertical Refreshing Scanning (VRS) is supplied. Mode 6 is used as the quick-scan axis and is called HFS in raster scanning; DACs mentioned in structure description of the electromagnetic micromirror is used as the slow-scan axis and is called VRS in raster scanning. The analysis results show that the slow axis participates in many resonant motions under the signals of resonant frequencies (Figure 3 and Table 2). Combined with the FEM (Figure 2), in VRS control, when the slow-scan axis operates in a noisy environment with resonant frequencies shown in Table 2, ten resonant motions are inspired to destroy raster scanning, which means that the key to stable control of electromagnetic micromirror is the stability of 
TABLE 1: Main properties of electromagnetic micromirror.

\begin{tabular}{lccc}
\hline Mirror material & Effective mirror size $\left(\mathrm{mm}^{2}\right)$ & Coil material & Coil number \\
\hline $\mathrm{Si}$ & $1.2 \times 1.1$ & $\mathrm{Au}$ & 10 \\
\hline
\end{tabular}

\begin{tabular}{|l}
33469 max \\
29751 \\
-26032 \\
-22313 \\
18594 \\
14875 \\
11156 \\
7437.6 \\
3718.8 \\
0 min
\end{tabular}

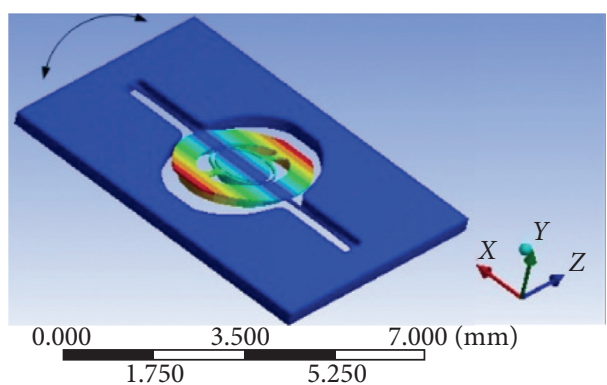

(a)

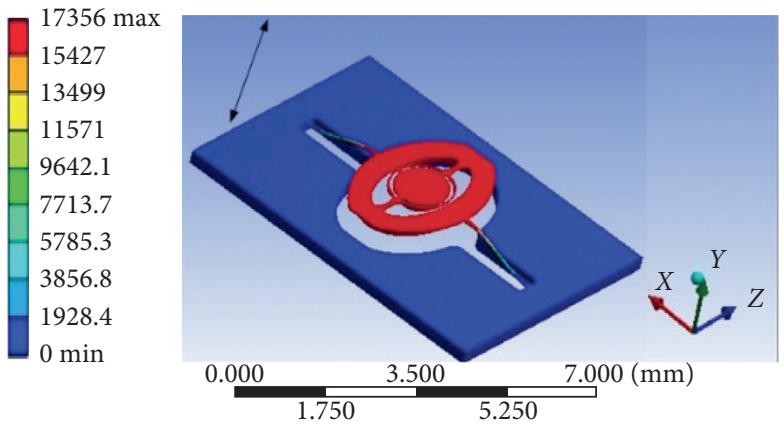

(c)

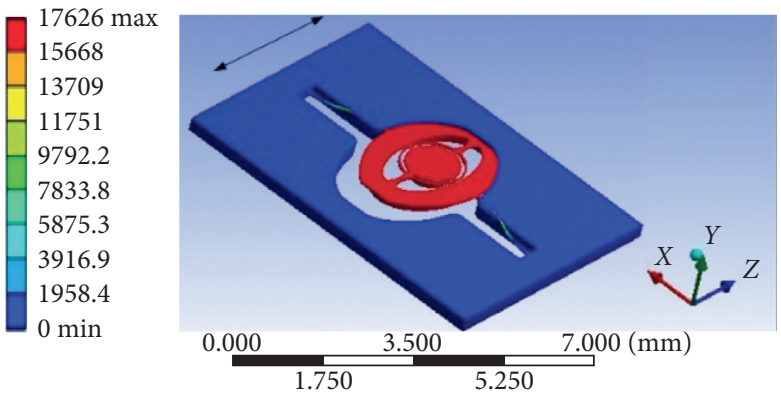

(e)
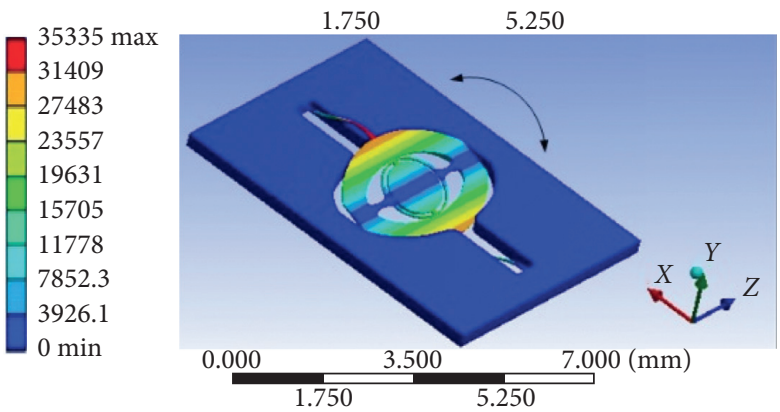

(g)

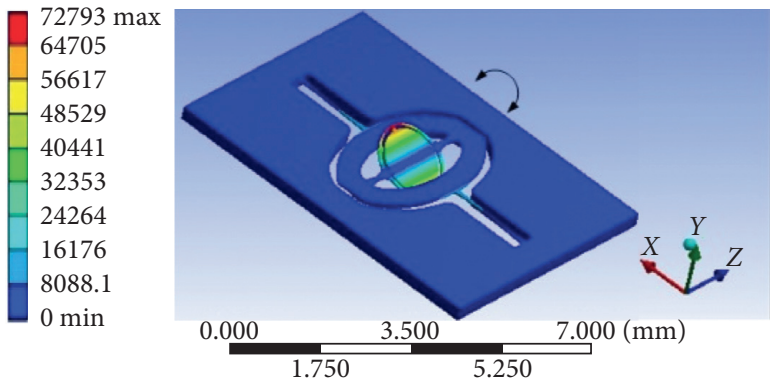

(b)

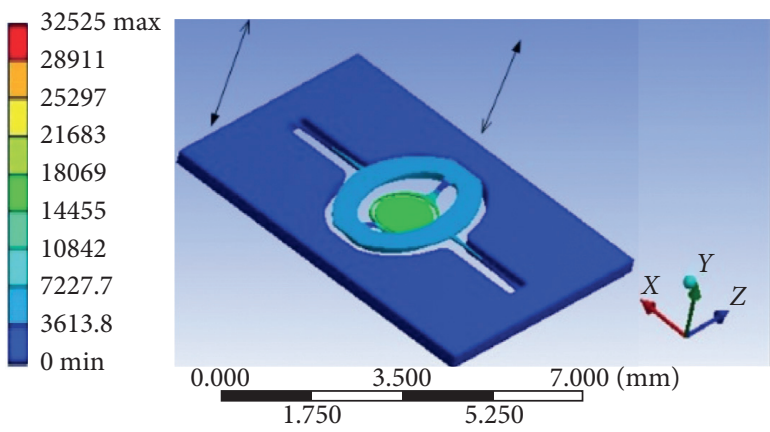

(d)

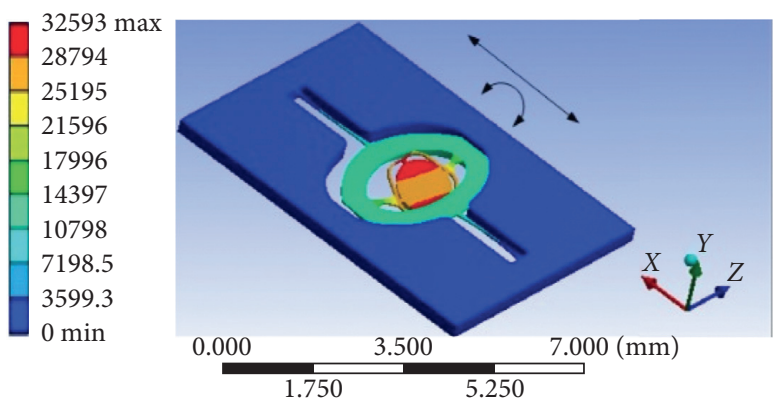

(f)

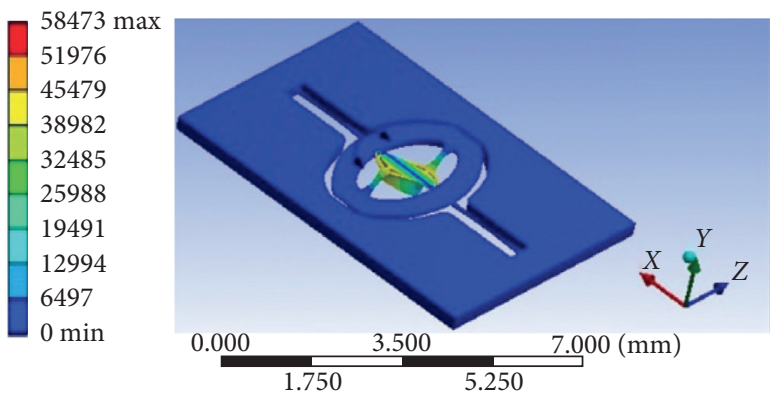

(h)

Figure 2: Continued. 


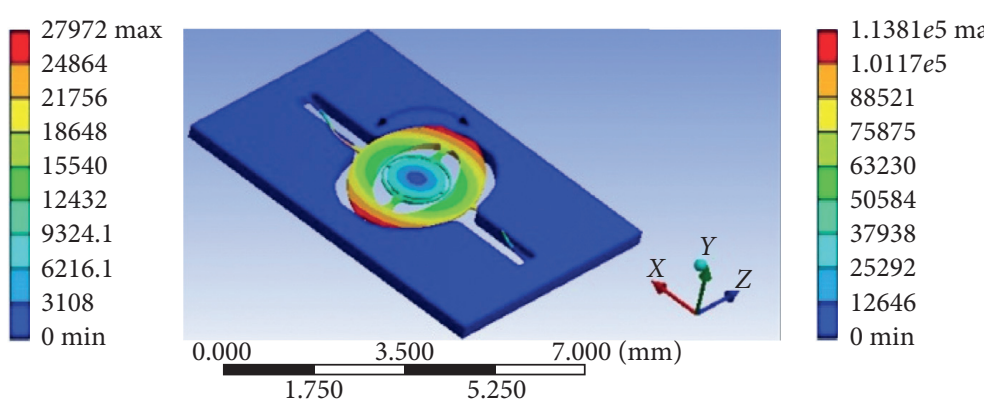

(i)

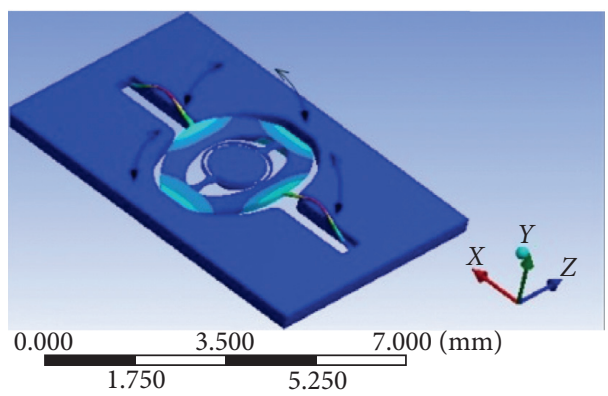

(j)

Figure 2: Ten resonant modes of electromagnetic micromirror. (a) Mode 1. (b) Mode 2. (c) Mode 3. (d) Mode 4. (e) Mode 5. (f) Mode 6. (g) Mode 7. (h) Mode 8. (i) Mode 9. (j) Mode 10.

TABLE 2: Characters of modal analysis.

\begin{tabular}{|c|c|c|}
\hline Modes & $\begin{array}{l}\text { Resonant frequencies } \\
\qquad(\mathrm{Hz})\end{array}$ & Motions \\
\hline Mode 1 & 770.97 & $\begin{array}{l}\text { Inner frame, slow beams, ellipse micromirror, ellipse reinforce ring, quick beams, elliptical ring } 1 \text {, and } \\
\text { elliptical ring } 2 \text { were taken as a whole (W1) and rotate around slow beams }\end{array}$ \\
\hline Mode 2 & 1523.9 & Motion of W1 is perpendicular to the outer frame plane \\
\hline Mode 3 & 2786.6 & Motion of W1 is horizontal in the outer frame plane and is perpendicular to original slow beams \\
\hline Mode 4 & 5150.2 & W1 is rocking around the quick beams \\
\hline Mode 5 & 5487.9 & W1 revolves around its center in outer frame plane \\
\hline Mode 6 & 16309 & $\begin{array}{c}\text { Ellipse micromirror, ellipse reinforce ring, quick beams, and elliptical ring } 1 \text { taken as a whole (W2) } \\
\text { rotate around the quick beams }\end{array}$ \\
\hline Mode 7 & 21711 & $\begin{array}{c}\text { W2 and inner frame with slow beams are vertical motions which are perpendicular to the outer frame, } \\
\text { while their directions are opposite }\end{array}$ \\
\hline Mode 8 & 50074 & $\begin{array}{l}\text { Motion of inner frame is horizontal along the original slow beams, while W2 rotates around quick } \\
\text { beams and ellipse reinforce ring may cause some distortions which are not recovered }\end{array}$ \\
\hline Mode 9 & 52054 & W2 rotates around the slow beams \\
\hline $\begin{array}{l}\text { Mode } \\
10\end{array}$ & 62000 & $\begin{array}{l}\text { Edges along the slow beams and edges along the quick beams both in the inner frame are folding and } \\
\text { unfolding at the same time, while in the opposite direction they may cause some distortions which are } \\
\text { not expected }\end{array}$ \\
\hline
\end{tabular}

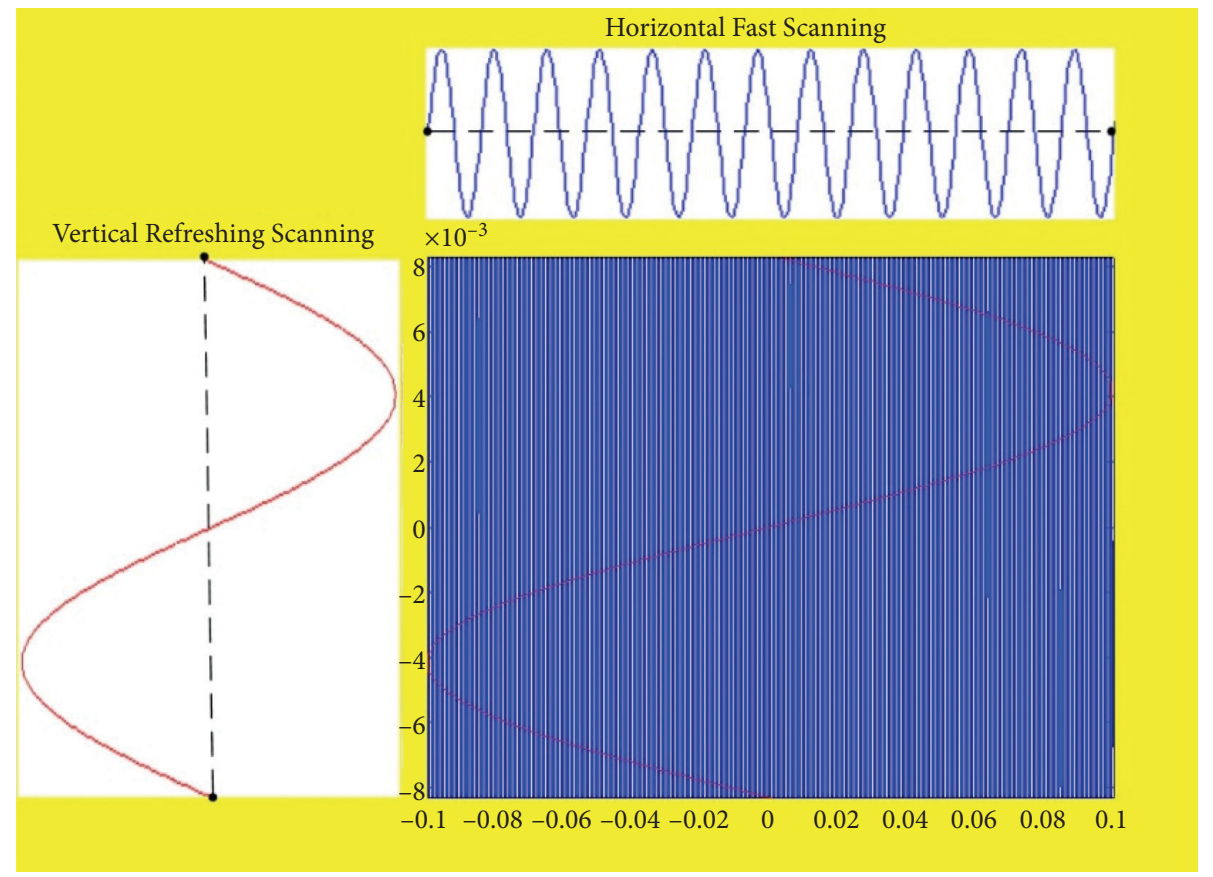

FIGURE 3: Principle of raster scanning. 
controlling slow-scan axis. In this research, slow-scan axis control is focused on, and filters of the slow-scan axis are designed to give a solution to this issue.

\section{Filters Design}

Although there are many noise removal methods such as $\mathrm{m}$-derived filtering, Butterworth filters, and elliptical filters [26], two issues of filtering for electromagnetic micromirror in this paper should be briefly discussed. One is that the designed filtering must effectively eliminate noise with frequencies close to that in ten modes to avoid vibrations of the electromagnetic micromirror. In particular, environment noise of frequencies near mode 1 should be filtered to ensure the reliability of slow axis control in laser scanning. Another is that the effectiveness of the signal must be ensured under no phase distortion to realize closed-loop control in slow-scan axis control for electromagnetic micromirror better. So, secondary filtering is developed. First filtering, equal-ripple low-pass filtering, would filter these noises with resonating frequencies effectively. Second filtering, zero-phase filtering, would preserve the features of the feedback signal at the exact location where they occur and would avoid a phase distortion.

In slow-scan axis control, noise with frequencies close to the resonant frequencies in ten resonant modes can inspire unexpected vibrations of the electromagnetic micromirror (Table 2). The actual resonant frequency $f_{\text {acl }}$ of electromagnetic micromirror in Mode 1 is derived from the ideal resonant frequency $f_{\mathrm{id} 1}=770.97 \mathrm{~Hz}$ (see also Figure 2) as

$$
f_{\mathrm{acl}}=Q_{1} f_{\mathrm{id} 1},
$$

where the scaling factor (the ratio between the actual resonant frequency and the ideal resonant frequency) $Q_{1}=$ 0.8432 in Mode 1 in this paper. It can be derived from (3) as follows:

$$
f_{\mathrm{acl}}=Q_{1} f_{\mathrm{id} 1}=0.8432 \times 770.97 \approx 650 \mathrm{~Hz}
$$

3.1. Equal-Ripple Low-Pass Filtering. Difference equation of Finite Impulse Response (FIR) filtering was described by Charles and Shenoi $[27,28]$ :

$$
y(i)=\sum_{i=0}^{n}(h(i) x(n-i)), \quad(i=0,1, \ldots, n),
$$

where $y(i)$ is the output, $x(n-i)$ are the inputs, $h(i)$ are the coefficients, and $n$ is the filtering order.

For slow-scan axis control in electromagnetic micromirror system, noise with frequencies close to resonant frequencies in ten modes can result in unexpected vibrations of the electromagnetic micromirror. Equal-ripple low-pass filtering, which has the advantage of controlling the boundary frequency strictly in the aiming frequency compared with other filters [29], is developed here. $y_{f}$ is the output of this equal-ripple low-pass filtering.

$H_{d}(\omega)$ is defined as the ideal magnitude of equal-ripple low-pass filtering, $H_{g}(\omega)$ is the actual magnitude, $N$ is the length of filtering, which is usually chosen as an odd number, and $n$ is the order of filtering; the weighted error function $W(\omega)$ is constant. Then, the error $E(\omega)$ between $H_{d}(\omega)$ and $H_{g}(\omega)$ can be expressed as follows:

$$
E(\omega)=W(\omega)\left|H_{d}(\omega)-H_{g}(\omega)\right|
$$

with

$$
\begin{aligned}
H_{g}(\omega) & =\sum_{i=0}^{M-1}(2 h(i) \cos (\omega i)+h(M) \cos (\omega M)), \\
N & =n+1 \\
M & =\frac{(N-1)}{2} .
\end{aligned}
$$

Combined with (A.14), the unit impulse response of the designed filtering satisfies

$$
h(i)=h(N-i-1), \quad(i=0,1, \ldots, n) .
$$

When electromagnetic micromirror operates in slowscan axis control with equal-ripple low-pass filtering, the constrained inequality about passband cut-off frequency $f_{p}$ and stopband cut-off frequency $f_{s}$ should be satisfied:

$$
f_{p}<f_{s}<f_{\text {acl }} .
$$

From (4) and (11), it becomes

$$
f_{p}<f_{s}<650 \mathrm{~Hz}
$$

Urey [9] reported that the slow-scan axis with the signal of the frequency $60 \mathrm{~Hz}$ and the quick-scan axis with the signal of the frequency $20 \mathrm{kHz}$, respectively, could support SVGA $(800 \times 600$ pixels $)$ for display. In this paper,

$$
60 \mathrm{~Hz}<650 \mathrm{~Hz} \text {. }
$$

So, sine signal with a frequency of $60 \mathrm{~Hz}$ is used to drive the slow-scan axis and noise with resonant frequencies in ten resonant modes should be filtered by equal-ripple low-pass filter. We set $f_{p}=60 \mathrm{~Hz}, f_{s}=61 \mathrm{~Hz}$. From (7), (10), and (A.15), we get the following:

$$
\begin{aligned}
E(\omega) & =W(\omega)\left|H_{d}(\omega)-H_{g}(\omega)\right| \\
& =W(\omega)\left|H_{d}(\omega)-\sum_{i=0}^{1}(2 h(i) \cos (\omega i)-h(2) \cos (2 \omega))\right| .
\end{aligned}
$$

Combined with Chebyshev Best Consistent Approximation (CBCA) [29, 30], $E(\omega)$ on equal-ripple low-pass filtering in domain $F=[0, \pi]$ could be represented as 


$$
\min \left[\max _{\omega \in F}|E(\omega)|\right]
$$

In the domain $F$, coefficients are chosen to make the maximum of $E(\omega)$ minimum.

Forward coefficient $h_{\text {eq }}$ of equal-ripple low-pass filtering on slow-scan axis control of electromagnetic micromirror, which is computed at Filter Design and Analysis (FDA) tool in Matlab (R2012a), is as follows:

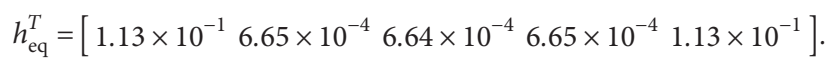

This is the first filtering.

3.2. Zero-Phase Filtering. For slow-scan axis of the electromagnetic micromirror, in order to preserve the features of feedback signal avoiding a phase distortion, the second filtering, zero-phase filtering, is developed, which would help preserve features appearing at the exact location to avoid the phase distortion [31]. Two traditional basic algorithms can express zero-phase filtering. One is FRR (Forward filter, Reverse filter, Reverse output), and the other is RRF (Reverse filter, Reverse filter, Forward output) [32]. Their principles are similar and the principle of FRR is given here:

$$
\begin{aligned}
& y_{1}(i)=y_{f}(i) * h(i), \quad(i=0,1, \ldots, n), \\
& y_{2}(i)=y_{1}(N-1-i), \quad(i=0,1, \ldots, n), \\
& y_{3}(i)=y_{2}(i) * h(i), \quad(i=0,1, \ldots, n), \\
& y_{s}(i)=y_{3}(N-1-i), \quad(i=0,1, \ldots, n) .
\end{aligned}
$$

In (17)-(20), $y_{f}(i)$ is the signal sequence from first filtering, $*$ is the convolution algorithm, $y_{i_{1}}(i),\left(i_{1}=1,2,3\right)$ are the output sequences after every transformation, $h(i)$ is the digital impulse response sequence, and $y_{s}(i)$ is the final output sequence after the FRR. In the frequency domain, (21)-(24) are deduced:

$$
\begin{aligned}
& Y_{1}\left(e^{j \omega}\right)=Y_{f}\left(e^{j \omega}\right) H\left(e^{j \omega}\right), \\
& Y_{2}\left(e^{j \omega}\right)=e^{-j \omega(N-1)} Y_{1}\left(e^{-j \omega}\right), \\
& Y_{3}\left(e^{j \omega}\right)=Y_{2}\left(e^{j \omega}\right) H\left(e^{j \omega}\right), \\
& Y_{s}\left(e^{j \omega}\right)=e^{-j \omega(N-1)} Y_{3}\left(e^{-j \omega}\right) .
\end{aligned}
$$

Referencing (B.1) and (B.2),

$$
Y_{s}\left(e^{j \omega}\right)=Y_{f}\left(e^{j \omega}\right)\left|H\left(e^{j \omega}\right)\right|^{2}
$$

In (25), there is zero phase between $Y_{s}\left(e^{j \omega}\right)$ and $Y_{f}\left(e^{j \omega}\right)$. This is the second filtering.

3.3. Experiments on Filters. Experiments and validations completed on platform (see Section 4) on these filters are shown in Figures 4 and 5.
Figure 4 shows the slow-scan axis response from collecting circuit after equal-ripple low-pass filtering filtered by zero-phase filtering under external disturbance, especially under electromagnetic interference similar to cell phone calls. Equal-ripple low-pass filtering effectively eliminates the vibration caused by environmental noise with the frequency close to the resonant frequency $650 \mathrm{~Hz}$ in Mode 1 (see the third rows in Figure 4). After the equal-ripple lowpass filtering, only the signal with the frequency of $60 \mathrm{~Hz}$ is left, which is the same frequency of the input sine signal (see the first three rows in Figure 5), but this signal appears hairy (see the third row in Figure 4). Zero-phase filtering decreases this hairy appearance, especially in peaks and troughs (see the fourth row in Figure 4). Meanwhile, it preserves the frequency (see the fourth row in Figure 5). The forward coefficient $h_{\mathrm{ze}}$ of zero-phase filtering is $h_{\mathrm{ze}}^{T}=\left[\begin{array}{lllll}0.4 & 0.4 & 0.4 & 0.4 & 0.4\end{array}\right]$.

In a word, the first filtering, equal-ripple low-pass filtering, succeeds in filtering the signal with resonating frequency for slow-scan axis control of electromagnetic micromirror effectively, and the second filtering, zerophase filtering, succeeds in preserving the features of feedback signal after equal-ripple low-pass filtering at the original location where they occurred as soon as possible.

\section{Piecewise PID Control and Experiments}

Feedback control of MEMS is complicated due to the limited availability of sensor and disturbing characteristics on MEMS device. In this paper, FTPS is used to acquire the output signal to indirectly detect the angle of slow-scan axis [1], and the resolution of FTPS on the end of slow axis in VRS control is $1 V @ 3 \mathrm{deg}$. Slow axis operates in open loop control with input sine signal voltage of a frequency $60 \mathrm{~Hz}$ and an amplitude $2 \mathrm{~V}$, respectively. The maximum absolute angle error $0.67^{\circ}$ appears in sines peaks and troughs (shown in Figure 6). Discrete PID control with filters design is firstly considered to be utilized for angle error reduction in slow-scan axis control. Its algorithm is presented by

$$
u(k)=K_{p} e(k)+K_{i} \sum_{j_{2}=0}^{k} e\left(j_{2}\right)+K_{d}[e(k)-e(k-1)]
$$

with an integral gain $K_{i}$ :

$$
K_{i}=\frac{\left(K_{p} T\right)}{T_{I}}
$$

and a derivative gain $K_{d}$ :

$$
K_{d}=\frac{\left(K_{p} T_{D}\right)}{T}
$$

where $T$ is a sampling time, $e(k)$ is a current error at $k$ point, $e(k-1)$ is a previous error at $k-1$ point, $K_{p}$ is a proportional gain, and $e(0)$ is set as 0 . 

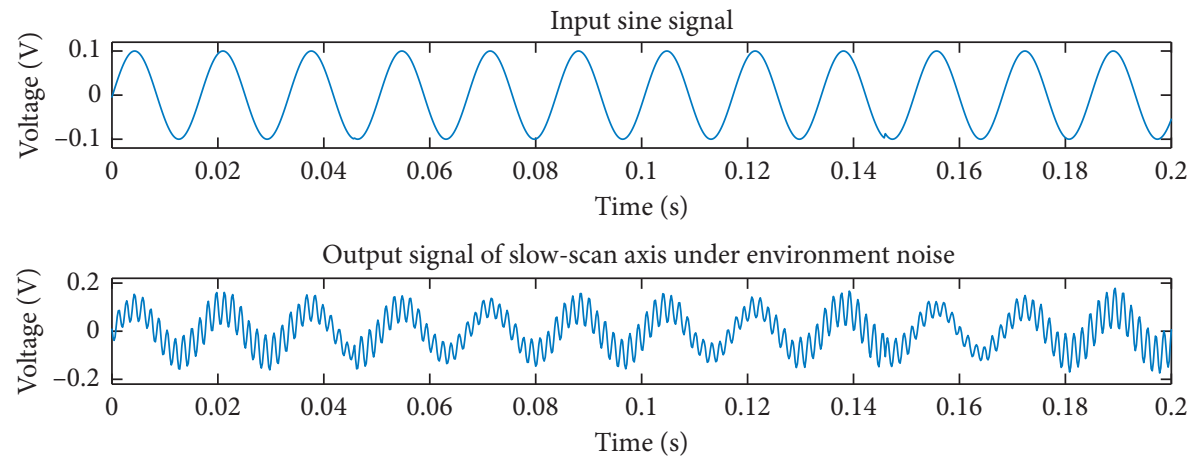

Output signal of slow-scan axis under environment noise filtered by equal-ripple low-pass filtering

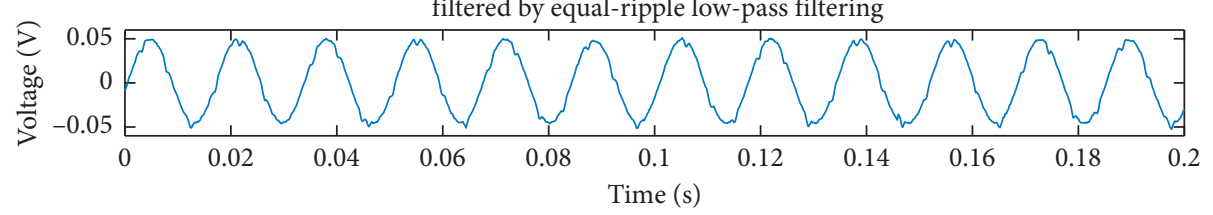

Output signal of slow-scan axis under environment noise after

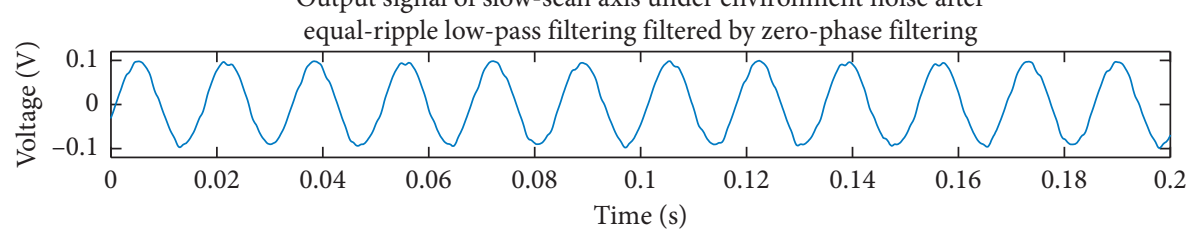

FIGURE 4: Validation of filters in aspect of signal.
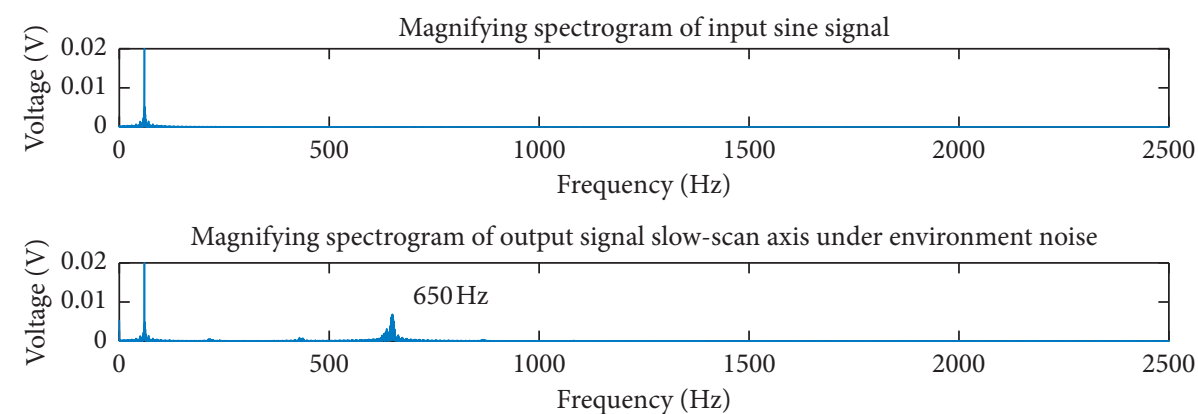

Magnifying spectrogram of output signal of slow-scan axis under

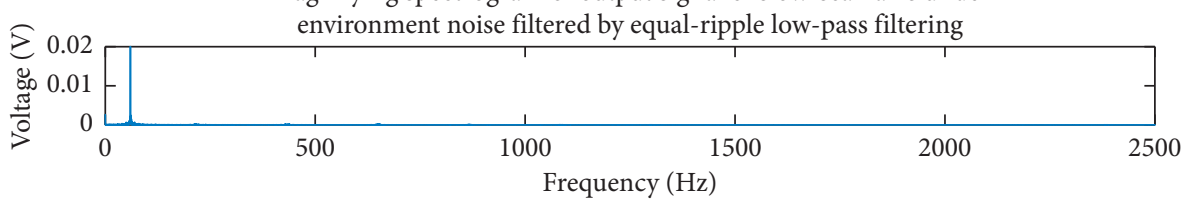

Magnifying spectrogram of output signal of slow-scan axis under environment

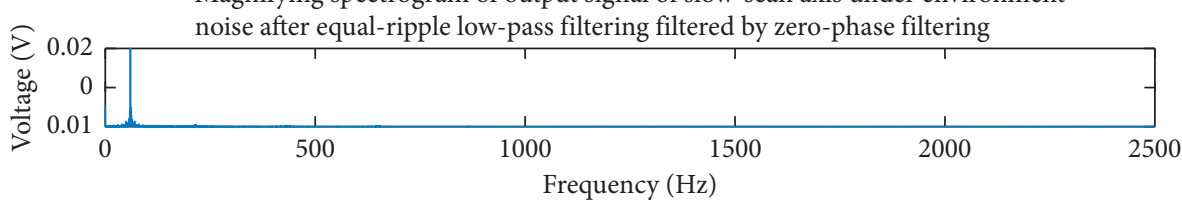

FIGURE 5: Validation of filters in aspect of spectrogram. 

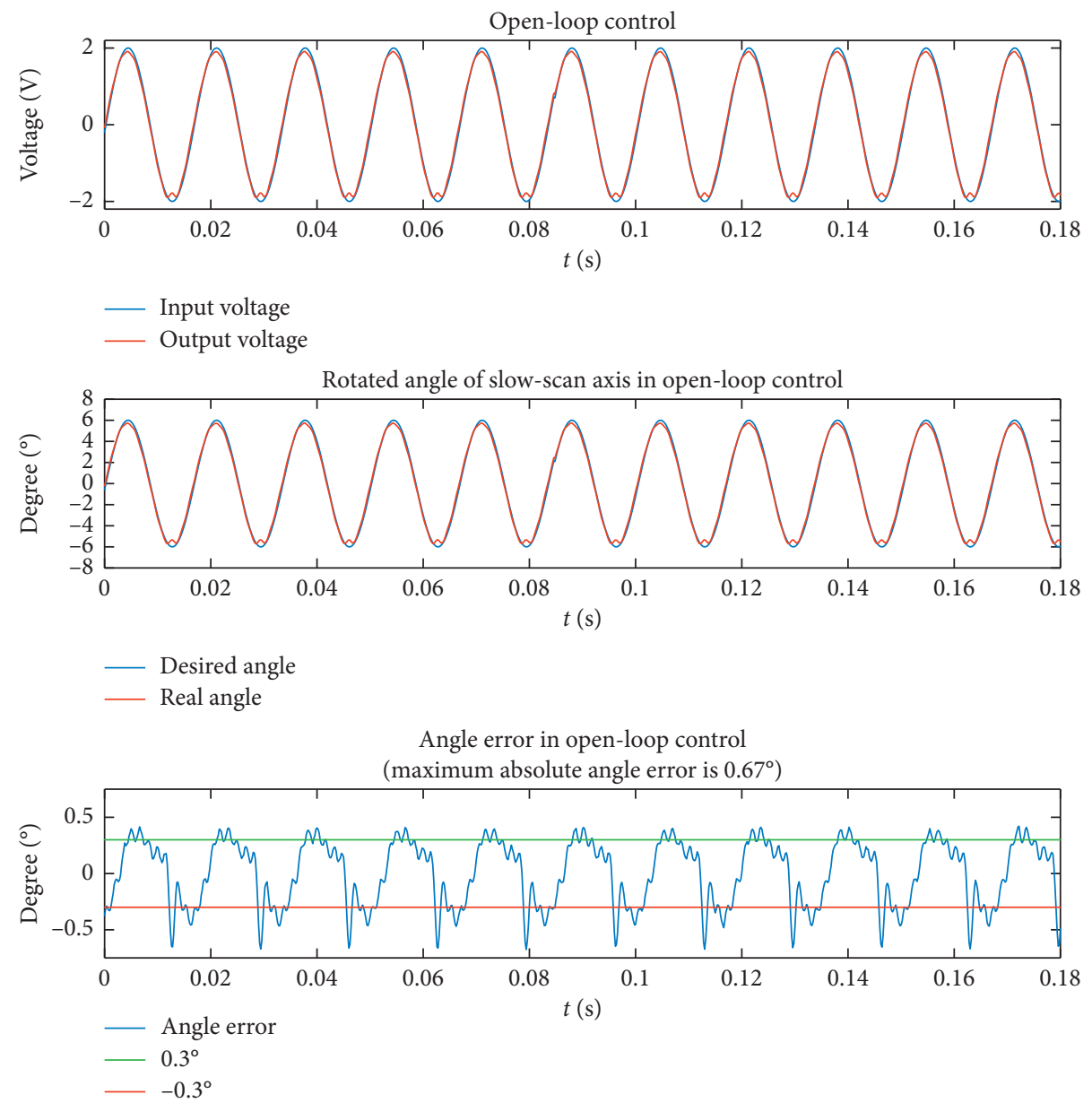

Figure 6: Slow-scan axis control of electromagnetic micromirror in open-loop control.

Figure 7 shows its control results. Indirectly measured real angle from feedback voltage can track the desired angle to some extent (in the 2 nd and 3 rd row of Figure 7). The maximum absolute angle error in sines peaks and troughs is reduced, compared with that in open loop control (Figure 6). But this maximum absolute angle error is still big. Here, $K_{p}=3.685, \quad K_{i}=0.002144$, and $K_{d}=35.175$, respectively.

Due to the accumulation in an integral part $K_{i} \sum_{j_{2}=0}^{k} e\left(j_{2}\right)$, the angle error may be bigger for slow-scan axis control of electromagnetic micromirror in discrete PID control. Therefore, a discrete piecewise PID control with filters design is developed and its algorithm can be denoted as follows:

$$
u(k)=K_{p} e(k)+\beta K_{i} \sum_{j_{2}=0}^{k} e\left(j_{2}\right)+K_{d}[e(k)-e(k-1)],
$$

with a switching coefficient $\beta$ :

$$
\beta= \begin{cases}1, & |e(k)| \leq e_{0}, \\ 0, & |e(k)|>e_{0} .\end{cases}
$$

Figure 8 shows its structure diagram in slow-scan axis control. When $|e(k)|>e_{0}$, discrete Proportional Derivative (PD) control is adopted to avoid the accumulation of integral part to reduce the angle error; when $|e(k)| \leq e_{0}$, discrete PID control is used to achieve the desired angle of slow-scan axis for the electromagnetic micromirror. Figure 9 shows that its control advantage is that the maximum absolute error is limited in $0.30^{\circ}$.

$$
\text { Notably, } \beta=\left\{\begin{array}{ll}
1 & |e(k)| \leq e_{0} \\
0 & |e(k)|>e_{0}
\end{array}, e_{0}=0.009\right. \text {, and sampling }
$$
frequency $F_{S}$ in experiments is $5 \mathrm{kHz}$. Screens were captured, when the slow-scan axis was in open control, in discrete PID control, and in discrete piecewise PID control, respectively. The ringing effect occurred in open control seen as in Figure 10(a). A tiny fraction of this ringing effect was 

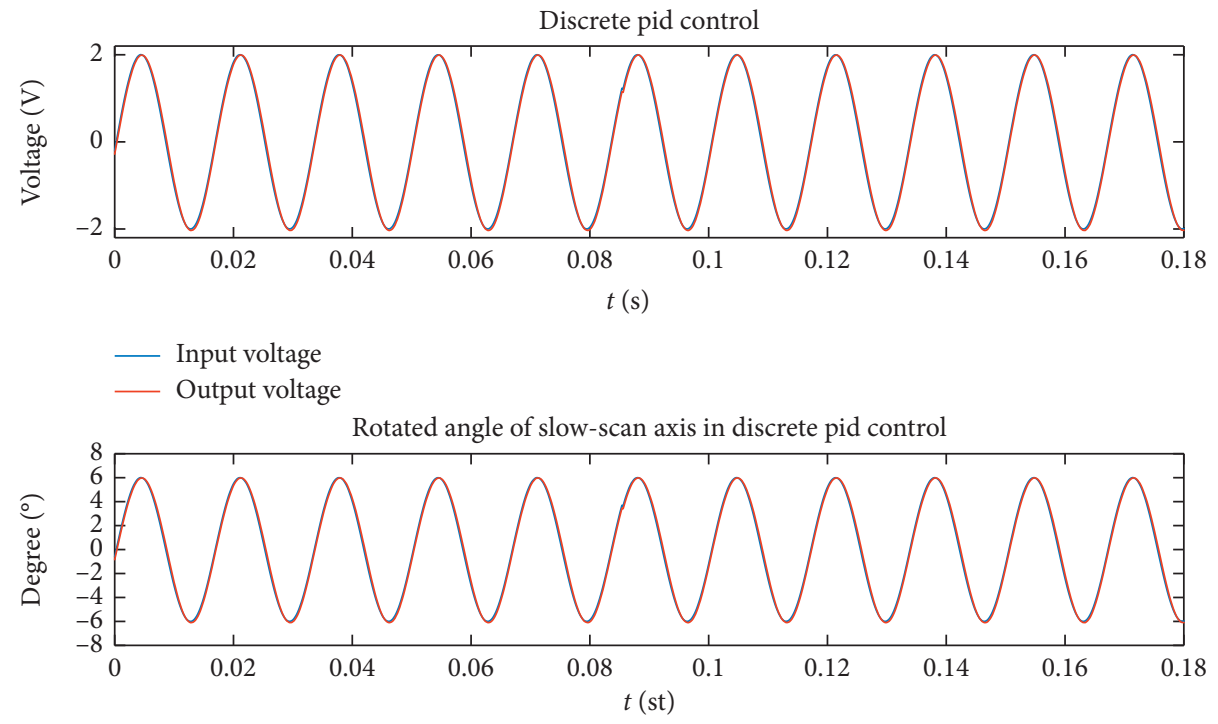

- Desired angle

- Real angle

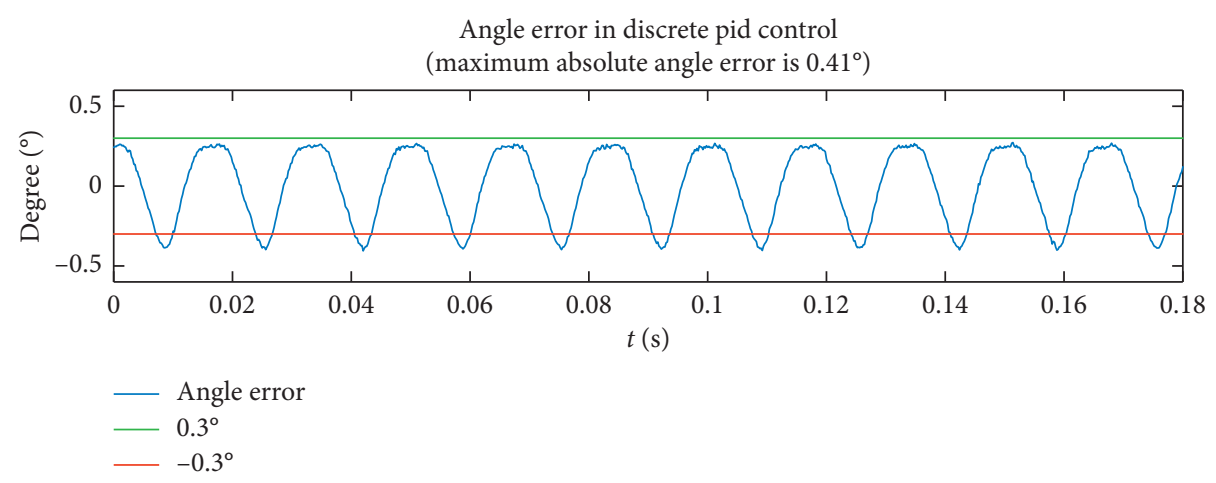

FIgURE 7: Slow-scan axis control of electromagnetic micromirror in discrete PID control.

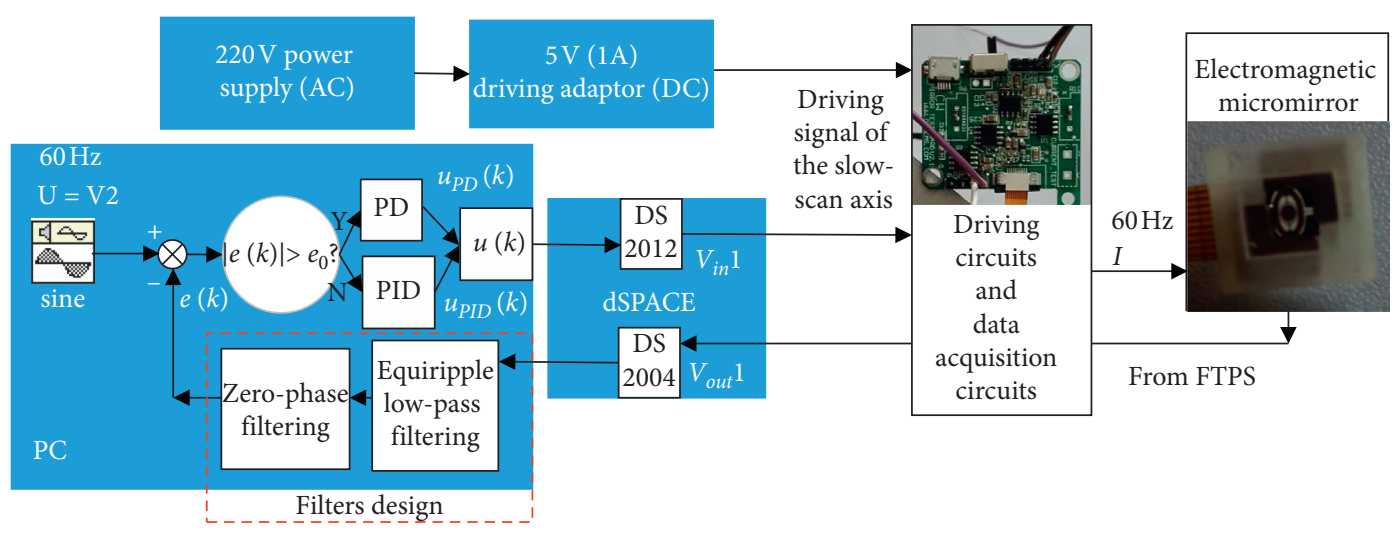

Figure 8: Structure diagram in slow-scan axis control.

removed in PID control as shown in Figure 10(b). Substantial amount of ringing effect was removed in discrete piecewise PID control (Figure 10(c)).
Figure 11 shows the raster scanning realized by the single red laser beam projected perpendicularly to this electromagnetic micromirror of two dimensions and 

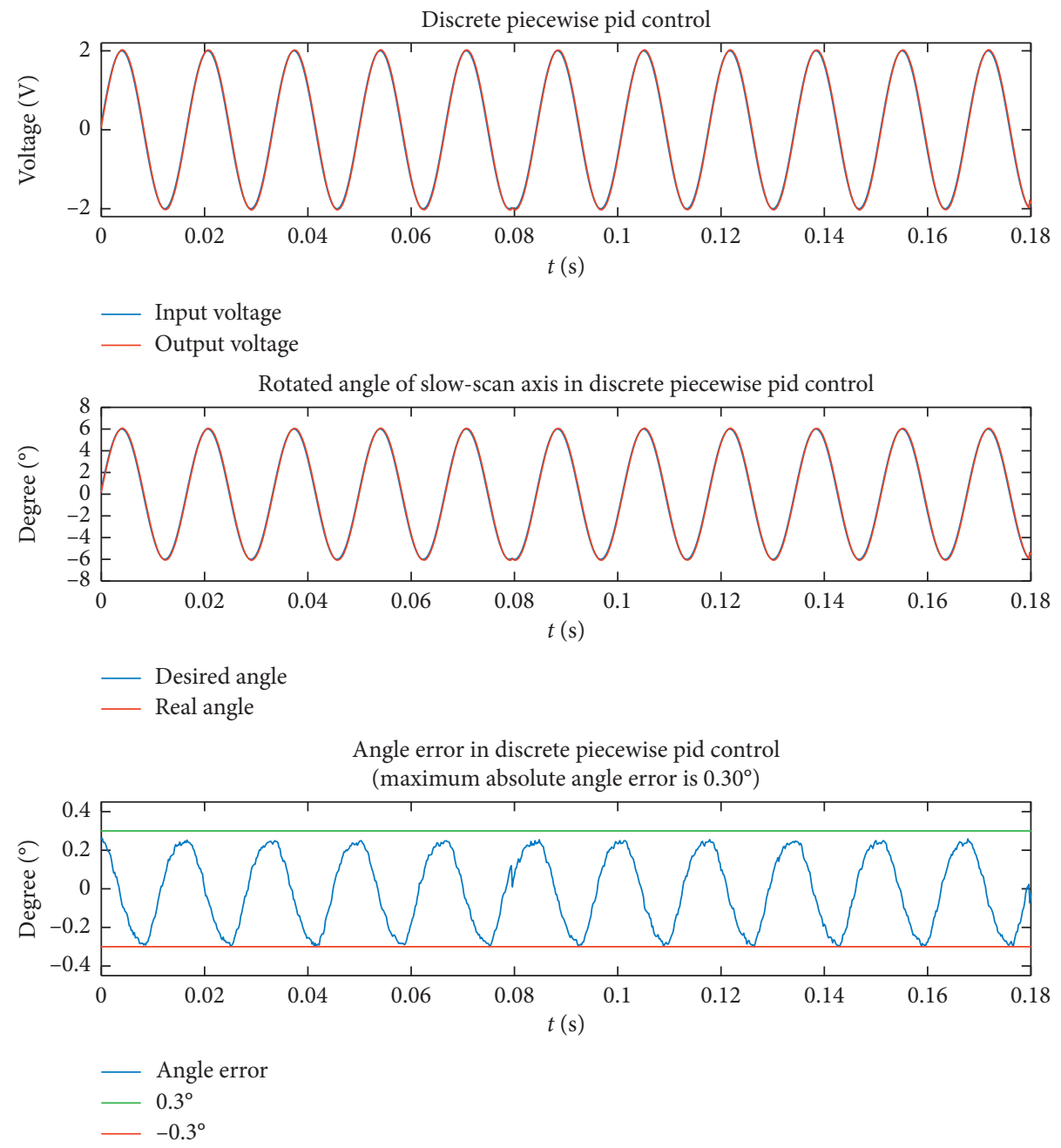

FIgURE 9: Slow-scan axis control of electromagnetic micromirror in discrete piecewise PID control.

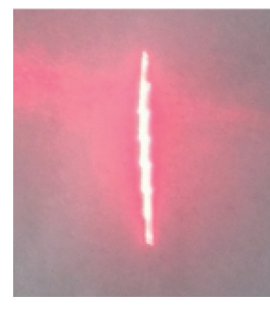

(a)

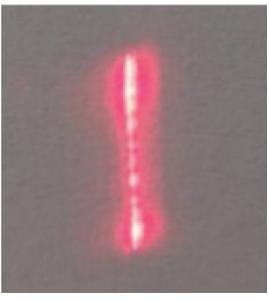

(b)

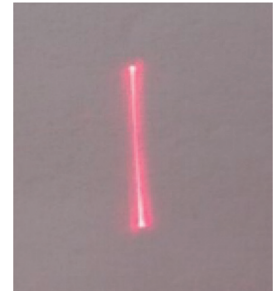

(c)

FIGURE 10: Screens captured in open control, in discrete PID control, and in discrete piecewise PID control. (a) Screen captured in open control; (b) screen captured in discrete PID control; (c) screen captured in discrete piecewise PID control.

reflected to the screen. In Figure 11, the slow axis operates in piecewise PID control; meanwhile, the quick axis is harmonically driven by sine signal with the resonant frequency
$22.113 \mathrm{kHz}$ and the amplitude $1.8 \mathrm{~V}$ supplied by the function waveform generator (Agilent 33250A). This raster scanning pixel resolution is SVGA $(800 \times 600$ pixels $)$. 


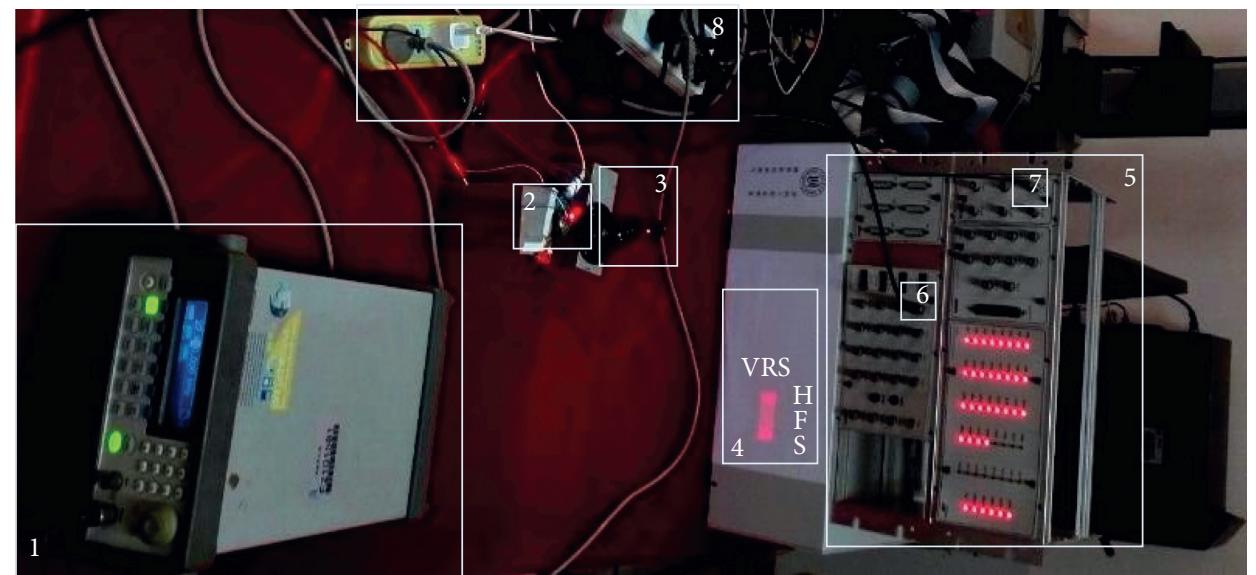

FiguRE 11: Raster scanning realized by the electromagnetic micromirror of two dimensions: 1. function waveform generator (Agilent 33250A); 2. electromagnetic micromirror with FTPS, driving circuits, and data acquisition circuits; 3. red laser; 4. screen; 5. dSPACE and PC; 6. $V_{\text {in }} 1 ; 7 . V_{\text {out }} 1 ; 8$. power.

\section{Conclusions}

In this paper, motions of electromagnetic micromirror succeed in being analyzed through FEM. Mode 1 is emphasized to be eliminated to improve the stability in the slow-scan axis control of raster scanning. The discrete piecewise PID control with secondary filters put forward here was efficiently verified by experiment to remove the ringing effect caused by the instability of slow-scan control.

\section{Appendix}

\section{A. Values of $n, M$, and $N$}

Kaiser proposed a formula of approximating $n$ as follows:

$$
n=\frac{-20 \lg \left(\xi_{1} \xi_{2}\right)-13}{14.6 \Delta f}
$$

with

$$
\begin{aligned}
& \Delta f=\frac{\omega_{s}-\omega_{p}}{2 \pi}, \\
& \omega_{p}=\frac{2 \pi f_{p}}{F_{S}}, \\
& \omega_{s}=\frac{2 \pi f_{s}}{F_{S}}, \\
& \xi_{1}=\frac{10^{\left(a_{p} / 20\right)}-1}{10^{\left(a_{p} / 20\right)}+1} \\
& \xi_{2}=10^{-\left(a_{s} / 20\right)},
\end{aligned}
$$

where $f_{p}$ is the passband cut-off frequency, $f_{s}$ is the stopband cut-off frequency, $a_{p}$ is the maximum passband attenuation, $a_{s}$ is the minimum stopband attenuation, $\xi_{1}$ is the ripple of the passband, $\xi_{2}$ is the ripple of the stopband, and $F_{S}$ is the sampling frequency. We set $f_{p}=60 \mathrm{~Hz}$, $f_{s}=61 \mathrm{~Hz}, a_{p}=3.5$, and $a_{s}=12$ and combining with equations (A.3)-(A.6) and $F_{S}=5 \mathrm{kHz}$ mentioned in experiments, we have

$$
\begin{aligned}
& \omega_{p}=\frac{2 \pi f_{p}}{F_{S}}=\frac{2 \pi \times 60}{5000}=7.54 \times 10^{-2} \\
& \omega_{s}=\frac{2 \pi f_{s}}{F_{S}}=\frac{2 \pi \times 61}{5000}=7.67 \times 10^{-2} \\
& \begin{aligned}
\omega_{c} & =\frac{\omega_{p}+\omega_{s}}{2}=\frac{7.54 \times 10^{-2}+7.67 \times 10^{-2}}{2} \\
& =7.605 \times 10^{-2}, \\
\Delta f & =\frac{\omega_{s}-\omega_{p}}{2 \pi}=\frac{7.67 \times 10^{-2}-7.54 \times 10^{-2}}{2 \pi} \approx 2 \times 10^{-4} \\
\xi_{1} & =\frac{10^{\left(a_{p} / 20\right)}-1}{10^{\left(a_{p} / 20\right)}+1}=\frac{10^{(3.5 / 20)}-1}{10^{(3.5 / 20)}+1} \approx 0.2, \\
\xi_{2} & =10^{-\left(a_{s} / 20\right)}=10^{-(12 / 20)}=0.25 \\
n & =\frac{-20 \lg \left(\xi_{1} \xi_{2}\right)-13}{14.6 \Delta f}=\frac{-20 \lg (0.2 \times 0.25)-13}{14.6 \times 2 \times 10^{-4}} \approx 4.10
\end{aligned}
\end{aligned}
$$

Substituting (A.13) into equation (8) in Section 3.1 yields

$$
\begin{aligned}
& N=n+1=4+1=5, \\
& M=\frac{(N-1)}{2}=\frac{(5-1)}{2}=2 .
\end{aligned}
$$




\section{B. Derivation on Principle of Zero- phase Filtering}

Combined with equations (10), (17)-(20), (B.1) is deduced:

$$
\begin{aligned}
y_{s}(i) & =y_{3}(N-1-i)=y_{2}(N-1-i) * h(N-1-i) \\
& =y_{2}(N-1-i) * h(i)=y_{1}(N-1-(N-1-i)) * h(i) \\
& =y_{1}(i) * h(i)=y_{f}(i) * h(i) * h(i), \quad(i=0,1, \ldots, n) .
\end{aligned}
$$

So in the frequency domain, it yields

$$
Y_{s}\left(e^{j \omega}\right)=Y_{f}\left(e^{j \omega}\right)\left|H\left(e^{j \omega}\right)\right|\left|H\left(e^{j \omega}\right)\right|=Y_{f}\left(e^{j \omega}\right)\left|H\left(e^{j \omega}\right)\right|^{2} .
$$

\section{Data Availability}

Partial properties of electromagnetic micromirror on structure used in analysis are protected by patient privacy and cannot be opened, while requests for further information if necessary will be considered by authors upon request (tany@shnu.edu.cn or wjshen2011@sinano.ac.cn).

\section{Conflicts of Interest}

The authors declare no conflicts of interest.

\section{Acknowledgments}

Thanks are due to Huijun Yu, Muyu Chen, Hai Tan, and Yanfei Huang in Professor Shen's team and Shaoyan Wu and Zupeng Zhou in Professor Tan's team for their help in the studying of previous researches. This work was supported in part by the National Natural Science Foundation of China under Grant 61971120 and Grant 61671303 and in part by the project of the Science and Technology Commission of Shanghai under Grant nos. 18070503000 and 18PJ1400100.

\section{References}

[1] K. Yamada and T. Kuriyama, "A novel asymmetric silicon micro-mirror for optical beam scanning display," Sensors, Actuators, Machines and Systems, vol. 20, pp. 110-115, 1998.

[2] C.-D. Chen, Y.-J. Wang, and P. Chang, "A novel two-axis MEMS scanning mirror with a PZT actuator for laser scanning projection," Optics Express, vol. 20, no. 24, pp. 27003-27017, 2012.

[3] H. Schenka, D. Kunzea, and H. Laknera, "A resonantly excited 2D-micro-scanning- mirror with large deflection," Sensors and Actuators A: Physical, vol. 1, no. 89, pp. 104-111, 2001.

[4] T. Bifano, "MEMS deformable mirrors," Nature Photonics, vol. 5, no. 1, pp. 21-23, 2011.

[5] M. Chen, H. Yu, S. Guo, R. Xu, and W. Shen, "An electromagnetically-driven MEMS micromirror for laser projection," in Proceedings of the IEEE 10th International Conference on Nano/Micro Engineered and Molecular Systems (NEMS), pp. 605-607, London, UK, 2015.

[6] A. Han, A. R. Cho, S. Ju et al., "Biaxial vector-graphic scanning micromirror using radial magnetic field," in Proceedings of the IEEE 18th International Conference on Solid-State Sensors,
Actuators and Microsystems (TRANSDUCERS), pp. 843-846, New York, NY, USA, 2015.

[7] J. H. Kim, S. W. Lee, H. S. Jeong, S. K. Lee, C. H. Ji, and J. H. Park, "Electromagnetically actuated 2-axis scanning micromirror with large aperture and tilting angle for lidar applications," in Proceedings of the 2015 IEEE 18th International Conference on Solid State Sensors, actuators and microsystems (TRANSDUCERS), pp. 839-842, Berlin, Germany, 2015.

[8] M. Liang, "Performance characterization of a single bi-axial scanning MEMS mirror-based head-worn display," Proceedings of SPIE Optical Scanning, vol. 2002, pp. 82-91, 2002.

[9] H. Urey, "Torsional MEMS scanner design for high-resolution scanning display systems," Proceedings of SPIE Optical Scanning, vol. 2002, pp. 27-37, 2002.

[10] H. Urey, "DMD 101: introduction to digital micromirror device (DMD) technology," 2008.

[11] X. Chu, L. Ma, S. Yuan, M. Li, and L. Li, "Two-dimensional optical scanning of a piezoelectric cantilever actuator," Journal of Electroceramics, vol. 21, no. 1-4, pp. 774-777, 2008.

[12] Y. Yingshun Xu, J. Singh, T. Selvaratnam, and N. Nanguang Chen, "Two-axis gimbal-less electrothermal micromirror for large-angle circumferential scanning," IEEE Journal of Selected Topics in Quantum Electronics, vol. 15, no. 5, pp. 1432-1438, 2009.

[13] Texas Instruments, “DLP4710 0.47 1080p, DMD,” 2018.

[14] C.-H. Ji, S.-H. Ahn, and K.-C. Song, "Hyoung-Kil Yoon and Moongoo Choi, Dual-axis electromagnetic scanning micromirror using radial magnetic field," MEMS 2006 Istanbul, vol. 15, pp. 32-35, 2006.

[15] A. D. Yalcinkaya, H. Urey, D. Brown, T. Montague, and R. Sprague, "Two-axis electromagnetic microscanner for high resolution displays," Journal of Microelectromechanical Systems, vol. 15, no. 4, pp. 786-794, 2006.

[16] C.-H. Ji, M. Choi, S.-C. Kim, K.-C. Song, J.-U. Bu, and H.-J. Nam, "Electromagnetic two-dimensional scanner using radial magnetic field," Journal of Microelectromechanical Systems, vol. 16, no. 4, pp. 989-996, 2007.

[17] S. Holmes, C. C. Rollins, K. Margaret, and B. C. Rothaar, Mehrube Mehrubeoglu and Gregory Schneider, Scaning Mirror Control, New York, NY, USA, 2011.

[18] A. Han, A. R. Cho, S.-H. Ju, J.-U. Ahn, C.-H. Ji, and Ji ChangHyeon, "Electromagnetic biaxial vector scanner using radial magnetic field," Optics Express, vol. 24, no. 14, pp. 15813-15821, 2016.

[19] W. Shen and H. Yu, MEMS Magnetic Field System of Scanning Mirror, Springer, China, 2015.

[20] W. Shen and H. Yu, MEMS Magnetic Field System of Scanning Mirror, Springer, China, 2017.

[21] M. Chen, W. Shen, H. Yu, T. Wang, and R. Xu, Methods on Enhancing the Anti-deformation Capability of MEMS Micromirror, Springer, China, 2016.

[22] S. Wenjiang, H. Yu, and F. Li, Large-size MEMS Electromagnetic Micromirror and its encapsulation, Springer, China, 2018.

[23] M. Bao, W. Qi, and Y. Wang, "Geometric design rules of fourterminal gauge for pressure sensors," Sensors and Actuators, vol. 2, no. 18, pp. 149-156, 1989.

[24] R. Bansal, "Fundamentals of engineering electromagnetics," CRC Press, Taylor and Francis Group, vol. 162, 2006.

[25] A. V. Oppenheim, A. S. Willsky, and S. Hamid Nawab, Signals \& System, Prentice-Hall, Englewood Cliffs, NJ, USA, 2nd edition, 1997.

[26] B. D. O. Anderson and B. Moore and John, Optimal filtering, Prentice-Hall, Englewood Cliffs, NJ, USA, 1979. 
[27] C. Rader, "Dsp history-the rise and fall of recursive digital filters," IEEE Signal Processing Magazine, vol. 23, no. 6, pp. 46-49, 2006.

[28] B. A. Shenoi, Introduction to Digital Signal Processing and Filter Design, John Wiley \& Sons, River Street, NJ, USA, 2006.

[29] G. Li, "Research about Chebyshev best consistent approximation and error function characteristics," Journal of China West Normal University (Narural Science), vol. 28, pp. 253256, 2007.

[30] G. Mo and K. Liu, Function Approximation Method, Science Press, Beijing, China, 2003.

[31] T. K. Rawat, Digital Signal Processing, Oxford University Press India, London, UK, 2015

[32] Y. Ji, S. Qin, and B. Tang, "Digital filtering with zero phase error," Journal of Chongqing University (Natural Science Edition), vol. 23, pp. 4-7, 2000. 